\title{
On Some Essential Requirements for a Fruitful Consciousness Research ${ }^{*}$
}

\author{
Asok Kumar Mukhopadhyay \\ All India Institute of Medical Sciences, New Delhi, India
}

\begin{abstract}
Absence of input of new ideas and paucity of relevant assumption, research question and hypothesis are the reasons why consciousness has not yet found its place in any of the algorithms of science. By weaving several novel ideas in series, in parallel and at multiple levels overarching several disciplines, a distinct roadmap has been drawn for a dispassionate consciousness research. Several workable propositions in the paper, interdisciplinary in its true sense, might lead to opening of multiple new doors of science.
\end{abstract}

Keywords: matter, consciousness, mind, life-principle, phenomenology, brain, cell-psychology, psychology and physics

“Traveler there is no path! Path is made by walking”.

\section{Introduction}

One of the major leading causes of poor DALY (disability adjusted life-years) worldwide is mental illness. On the other side, one of the leading causes, which can change the civilization of humanity in general is the input from accomplished spiritual mystics. Both of the facts compel us to address aggressively the issue of consciousness and to respond comprehensively. The issue highlights the necessity for a fruitful consciousness research with theory, ethics, and aesthetics. However, the problem is where to start from and what should be the appropriate research question and research hypothesis.

Let us begin with the experience of singularity of consciousness. Consciousness has been said to be singular and, in fact, experientially it is singular. The plurality, as observed, is introduced by the presence of the mind and/or the brain! In other words, multiplicity begins with functioning of the mind/brain, through their expressive behavior and their difference with those of a fellow being. In absence of the mind or of the brain as a structure, as a process, as a functioning organ, or when one goes beyond the mind (supramental) or the brain (supracortical!) consciousness as a reality is one and is only one. Erwin Schrödinger is absolutely right here! Examine another experience: "Awareness is one while perceptions are many”. Plurality here is because of

\footnotetext{
*Acknowledgements: The paper has been read and criticized by Prof. R.M. Pandey, Ph.D., Head Dept. of Biostatistics, and Dr. D. Bhowmik, professor of Nephrology, All India Institute of Medical Sciences (AIIMS), Dr. Millard Wohl, Ph.D., Nuclear scientist, USA, Dr. R. P. Upadhyay, Ph.D., senior scientist in Indian Institute of Technology, Delhi, Dr. Z. H. Khan, M.D., the President, of Swaasthya Sanchar organization, author's Ph.D. students and senior Research Fellow, Pooja Pallavi, M.Sc., and Rizwana Quadri, M.Sc., author's daughter Dr. Tapasyapreeti Mukhopadhyay, MBBS, an intern as a fresh medical graduate in Lady Harding Medical College, New Delhi. The author acknowledges all of them for their thoughtful contribution. The author is also grateful to Mrs. Pooja Taneja for her secretarial assistance.

Asok Kumar Mukhopadhyay, M.D., Department of Laboratory Medicine, All India Institute of Medical Sciences.
} 
informational conditioning of "self". When the self is stripped off its conditioned properties, perception is awareness.

The whole universe, or multiple universe(s), and the system of multiple universe(s), the multiversity are often experienced as "consciousness and its contents". What are those contents? Contents to start with, are mind, self, life as life-principle, and information. All are non-local, meaning none of the four is irrevocably localized in space and time. With the help of mother mind, information comes out form (space, time) and energy. Transformation of this information-based energy to matter (dark matter) occurs in nature beyond Planck's scale of measurement while mater-to-energy transformation happens within Planck's scale of nature. Space, time, energy, and matter are "local" and so also information when it is using the matter/energy or space/time as its vehicle. Consciousness, mind, self, life-principle, and information are non-observable influential(s) which influence the behavior at observable level within Planck's scale of measurement (Mukhopadhyay, 2013).

We, the human being like any other life-form, stand at the "boundary” between nature beyond Planck's scale of measurement and the nature within Planck's scale. We, the human being probably as the highest kind of life-form can do science at the domain of nature within Planck's scale of measurement and engage in abstraction of nature-consciousness from the domain beyond Planck's scale. The so-called yoga, in this sense, is bringing abstraction into rationality and down into sensibility.

The issue is how with such consciousness one does science and how the absolutely abstract consciousness can be brought to scientific rationale and down to sensible measurable realm?

\section{The Problem}

From the introductory paragraph, it is easy to get a glimpse of why consciousness is not yet brought in the algorithm of science. Science grows with new ideas, new assumption, research questions, and research hypotheses followed by testing of the hypothesis. Next follows generalization of the result into a theory and predictions from the theory. When prediction comes true, the results are further documented at observable and sensible level as real facts. For developing the realm of what is supposed to be a science of/for consciousness there is little input of new ideas, few relevant assumptions as required to begin with, paucity of appropriate research questions and there is only few workable research hypotheses. These preliminary requirements, which are essential for a dispassionate investigation, have been missing from the set agenda of consciousness research.

In consciousness research what is generally observed is an effort to connect consciousness directly with either "matter", or neuron, from the standpoint of expertise and experience in respective discipline. For this reason, many researchers from the disciplines like quantum physics, cybernetics, chaos, linguistics, computer science and simulation, neurophysiology, artificial intelligence, and artificial life have recognized the possible role of consciousness in science, and built up respective limited capacity theory on the issue based on the explanatory gaps in their experimental observations and imaginative faculty. Most of them have cashed on the explanatory power of their theory. What is missed is the assessment of the "theory's predictive power", if any. Also, although all of us do science as a conscious being, there is hardly any introspection on where this consciousness can fit in any of the algorithms used in science. Nevertheless, by this mental leaning towards consciousness, many researchers have enriched their respective discipline and learned its intricate fencing and therefore, its explicit limit. 
The effort to connect matter with consciousness is based on the assumption that with the rules and principles of quantum physics, it is possible to explain everything and all about consciousness including its operation, constrains, and even its origin, has not overcome the problem of measurement. Similarly, the attempt to connect the theory of relativity with a limitless domain of nature has resulted in the problem of singularity and that for quantum field theory one is stuck with the problem of infinity. Another flaw in the approach to consciousness is to assume consciousness as only "neurocentric" and to connect the assembly of neurons in the brain with consciousness/conscious experience. This is supposedly based on the assumption that consciousness emerges either from extensive synaptic networking of information in a non-discrete (e.g., Dennett) or in a discrete (e.g., Crick, Edelman etc.) manner, or from the information hub at the microtubules (e.g., Hameroff, Penrose). In "neurocentric" consciousness research we are not able to penetrate the "hard problem" or qualia mechanics. In the context of science of information when we are yet to learn how to harness information from interstellar and intergalactic space, a proposition like consciousness could be a super-super-computer having super-super-mind like properties (e.g., Thomas Campbell, Daniel Dennett) is expected to yield little sensible progress towards consciousness. In absence of successful engineering of vacuum, the efforts like connecting complexity or "chaos" with "quantum”, quantum with "information” or phenomenology are unlikely to offer any road map towards a science of/for consciousness! The proposed connection between quantum gravity and consciousness has still not come out of the mystical shed! In fact, doable hypothesis on this issue is hardly seen which can practically farther consciousness research.

In the paragraphs that follow, we would try to formulate some research questions and research hypotheses based on some new ideas and assumptions, which appear at this stage as imagination and seem not even "sane" by some of the conventional researchers.

\section{The Tentative Scheme}

While what is consciousness seems a philosophical problem, the questions like what does consciousness do and how does it do so remain important scientific issues. Not the ontological and the axiological aspects of consciousness but the epistemological and the phenomenal aspects of consciousness seem more amenable to scientific investigation. Consciousness as it seems is phenomenal consciousness while consciousness as it does is causal consciousness. In consciousness revolution, Roger Sperry (1987) observed a different form of non-eliminable causal determinism, which would be responsible for paradigm shift in several disciplines of science. Seeming consciousness is incomplete without causal consciousness and causal consciousness remains incomplete without axiological and ontological consciousness. Investigation of nature is prerogative of scientists and if consciousness also has a nature, (a great "if" indeed!), a great assumption to begin with, then science can certainly take up this challenge for making a science for (epistemic) consciousness and a science of (ontological) consciousness. Ontological consciousness hides behind the veil of its nature, the nature that is not necessarily confined to what is measurable within Planck's scale. The idea of existence of nature outside Planck's scale of measurement itself is a radical one, may be unpalatable to begin with, however seems refreshingly new for those looking forward to an opening. The vision of multiple universe(s) leads us to nature beyond Planck's scale of measurement.

To work out the connection between consciousness and matter one requires inputs of new ideas and some solid assumptions to begin with. One of such assumptions is that between matter and consciousness, the prominent milestone is that of mind. Consciousness-mind-matter is the route to travel whether one takes a 
top-down or a bottom-up approach. In the bottom-up approach this notion is reflected in Henry P. Stapp's visionary statement in Quo Vadis Quantum Mechanics (2005), "What is the future of quantum theory? Where will it go from here? Quantum theory will go where it is most needed, which is into the effort to understand ourselves, and in particular the connection of our minds to our bodies”. Mind, consciousness, and matter are categorically different. How this category difference originates remains an important research question!

Let us look into the further details in this assumption. For analysis of this assumption, the research question would be how matter and mind are connected. Not an easy nut that can be cracked! The research hypothesis to this effect is that the matter is connected to the mind through operation of information. Present day engineers are engaged in information technology and only very few scientists (e.g., Paul Davies, Millard Wohl) have been thinking on a probable science of information. The issue of connection between matter and mind can be addressed adequately having had more knowledge on information as "entity", on its structure, geometry, and operation. This could be the beginning of hypothesis generation for consciousness research! In a similar vein, one might raise the research question how mind is connected with consciousness. The hypothesis, which can be put forward is that mind is connected with consciousness through operation of "life", may be as we observe it as life-form, "living state of matter", or as life-principle or, what Tibor Ganti (2003) calls "principles of life”! According to Schrodinger, "A living substance avoids passing to equilibrium. It feeds on negative entropy”. Manifest consciousness as could be observed by our limited capacity senses is seen only in which is recognized as alive. In the bottom-up approach, therefore, the artificial life seems more close to consciousness than artificial intelligent system. Also this closer proximity of "life" to consciousness is evident to a limited extent in the interlinked operation of informational molecules responsible for distributed consciousness within a living cell or how the cell membrane is connected with the genome through proteome-epigenome-genome spectrum (Chen et al., 2003). The emergence and the surge of the paradigm, "Life style and health" may be considered an indivisible part of consciousness revolution, where phenomena in life are used to connect the mind with consciousness to achieve coherence with the whole for prevention or cure of an illness or disease. On the darker side, the poverty-ridden life-conditions are associated with, in general, a poor quality of functioning of mind, which in such condition is having diminished cognitive ability. The poverty takes an unimaginably huge toll on the psychiatric health, costing nation's human resources and productivity. Low and middle-income countries, according to World Health Organization's report (retrieved from http://www.who.int/gho/mortality_burden_disease/en/index.html) contribute about $75 \%$ of the global burden of all neuropsychiatric disorders. Strength of mind comes from life-principle. Therefore, it could be grossly stated that a better quality of "life" adds strength to the mind. Better "life", in general, offers a healthier mind for the individual as well as for the mass.

On elaboration, therefore, we have replaced the original spectrum of consciousness-mind-matter with consciousness-life-mind-information-matter spectrum. In research, which is a bottom-up approach, the milestones would be as stated above. For a top-down approach, consciousness can connect with the mind directly or via "life", and the mind can connect to the matter directly or through information (see Figure 1). 


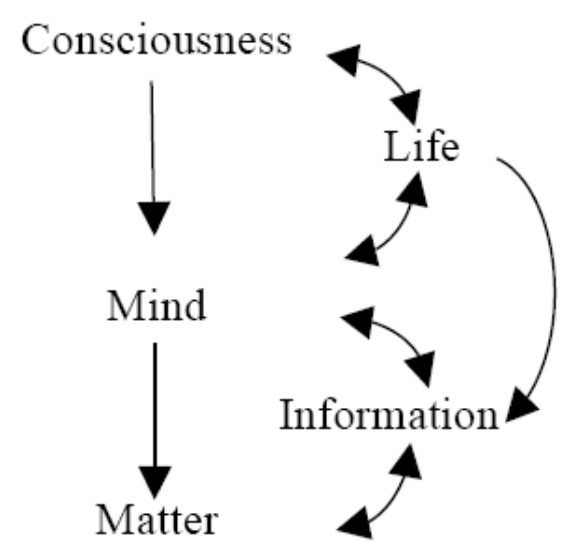

Figure 1. Hierarchical relationship between consciousness, mind, life, information, and matter.

\section{Tentative Time Frame}

In 19th century, we have learnt about material world to a large extent with the advent of classical physics. The 20th century brought to us the quantum physics with microscopic description of the material world. In 21st century let us do the physics of information. Science of information (Mukhopadhyay, 2013) is supposed to deal with its structure, geometry and operation, its relationship with genes, "memes", self, mind, consciousness, and the mechanism of its genesis and destruction. Science of mind is likely to grow and co-evolve in concurrence with science of information. In all probability we would be clearer about "life" in the 22nd century. There is expected to be concomitant growth of science of "self" in the direction of operation of corporeal self on memes, genes, and information, and on the technique of embodiment of "self" in intelligent system and robotics. Science of consciousness is expected to blossom fully in the 23rd century. We are, therefore, at the middle of five century's science. The proposed time frame is for mass consumption, letting sufficient time for further evolution of the human brain to acquire the ability of understanding consciousness (as cited in Colin McGinn's celebrated argument, "Just as monkeys cannot understand quantum theory, humans cannot understand consciousness"). Many scientists and philosophers, however, in their thought processes on consciousness are far ahead of their present time.

\section{Consciousness and Self}

Consciousness is absolutely abstract, diffuse, and without any boundary. Its concrete and particulate form within the boundary of the system is considered as "self". This is the self of any self-organizing system! As representative of consciousness, self is customized and conditioned with specific information to work within the given constrain of the system as the center of "irreducible subjectivity". The distinction between self and consciousness disappears when self surrenders its properties conditioned by information to consciousness. Unlike mind, "Self is categorically identical to the substance of consciousness".

That self is categorically identical to consciousness (consciousness is equivalent to God according to many religious beliefs) has some evidence-based support. In a recent neuroimaging study, Epley, Converse, Delbosc, Monteleone, and Cacioppo (2009) compared neural activation in subjects while reasoning about "will's of self, God and the 'average American'”. It was found that the contrast disappeared in subject who had been reasoning of self vs. God's will, unlike in subjects engaged in self vs. American's or God vs. American's will where two different stimulated areas were detected. 
Programmed by the "self", the mind processes information. While mind is sensitive to information, self is sensitive to phenomenon. Mind does not have the ability to experience and self experiences. Mind retains the memory of information (semantic memory). Self retains the memory of experience (episodic memory). To draw some examples for understanding the difference in functioning of self and mind, it can be said that a research fellow over four years or so, becomes a research scholar with organization of information and some practical experiences of how to prepare and submit a thesis for Ph.D. degree, while the supervisor mainly operates through his experience gathered in his "self". While the news of child abuse in a family or of the residents exposed to that of repeated terrorist attack at the national border are regarded as only information to the public, the children exposed to regular domestic violence or the residents facing the cross-border terrorism are often prone to develop personality disorders involving self. There is an ongoing debate whether depression, where there is a disconnection between the world and the self, is a result of the disease of the brain or is an illness of self. Balancing neurotransmitters with antidepressant medication might control the symptoms of depression but is unlikely to cure the disease! The whole immune system of the body works on the basis of distinction between "self" and non-self. Within the sub-system of lymphocytes and in many cells of parenchymal organs even the self has representative at the genetic and molecular level (e.g., major his to compatibility complex-I).

Self and mind have a combined product, the output of a joint venture known as intelligence. Self operates through mind and intelligence. The intelligence is externalized and mechanized within the matter to a very limited extent in an intelligent robot, which has mind-like information processing modules and a very limited self-like activity to take decision (as cited in SMT (the self-model theory of subjectivity) and PSM (the phenomenal self-model) of Thomas Metzinger (2007)). However, there is no element of "life" in the robot, and therefore, the robot cannot become self-conscious. There are many expert systems, which have access-consciousness, monitoring consciousness, and decision-making consciousness within the bounds of a large number of possibilities. However, no expert system developed so far has shown phenomenal consciousness, self-consciousness, or meta-self-consciousness. One can control an expert system through a remote, where the controlling self-consciousness of the remote-driver works for the robot.

\section{Elementary Phenomenology}

How is this self, known as the centre of phenomenology, created out of unbroken consciousness? What could be the phenomenology behind creation of self? Again, we may begin with an assumption! On careful analysis, it can be stated that during creation of self, which would be independent of, but would still continue to work in connection with unbroken consciousness, five phenomena are supposed to have a role to play. The relevant research question is what those elementary phenomena are. Probably, a joy of separation for gaining independence—-birth of new "life"; creating a new conditioned existence-“ego"; having a desire to create many like itself- "sex" in very elementary form; with a will to share the experience with others - the primordial manifestation of "love"; knowing sure of its limited time-span of independent existence following which it has to relinquish its independence-a phenomenon with elementary meaning of "death". Love, sex, life, death, and ego, therefore, are not just personal event but phenomena at the elementary level, which leave their imprint on self and form its five private facets that work as its motivational epitome. Our research hypothesis in phenomenology is, therefore, that this elementary phenomenology stands in between surface phenomenology of material (quantum) world and depth phenomenology of consciousness (see Figure 2). 


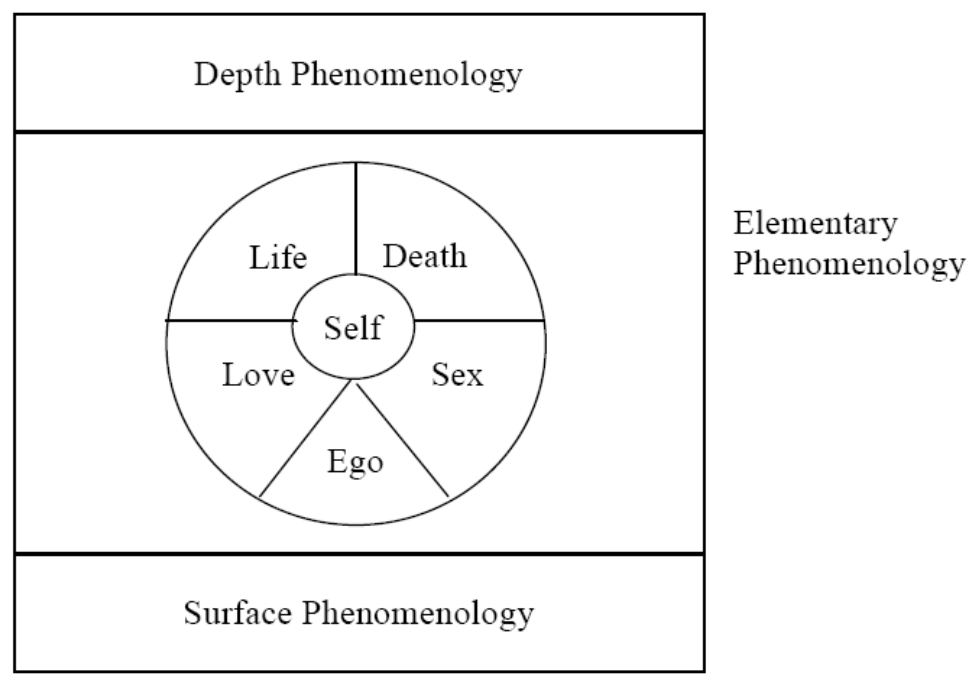

Figure 2. Depth, surface, and elementary phenomenology.

Thomas Nagel (1993) has been insisting on finding out the "elements" in conscious experience. He offers the analogy that as atoms and molecules are to chemical science, so the elemental conscious experience could be the ingredients for complex consciousness experience. And, Eric Kandel (2013) has endorsed Nagel's view. Therefore, another probable research frontier could be to examine these five phenomena at elementary experiential level with what Nagel has been looking for! At a practical level, a cardiologist might bring consciousness and elementary phenomenology in the algorithm of pathogenesis of ischemic heart disease with a research hypothesis that a deep conflict between two elementary phenomena, "love" and "ego" leads to coronary spasm. A psychologist may try to prove or falsify a hypothesis that divorce results from a conflict of "love", "sex", and "ego" between the married couple. The psychic origin of autoimmune disease could be traced to a turbulent phenomenology where someone who is supposed to protect the sanctity of self, has been repeatedly attacking its dignity (e.g., incest). Phenomenology and molecular medicine could be connected with a research hypothesis that love has a genetic basis. One cannot love music or a flower unless one has genes for it. Love turns on the genes. The molecular machinery of gene expression runs in tandem with phenomenology of love.

\section{Consciousness and the Brain}

Let us examine the other area where consciousness research is almost synonymous to research in the field of neuroscience. After the origin of a unicellular organism, it took one and a half billion years for a neuron to evolve. For evolution of the brain, it took another half billion years. Using the computer metaphor, several of the scientists consider the brain as hardware of which the equivalent software could be mind. Many philosophers go a step ahead to consider mind as hardware and consciousness as software. The brain, however, is not matter and is certainly not hardware in the sense we understand it. The brain consists of all living neurons, 10 (Kandel, 2013) in number along with 10 (Shomrat \& Levin, 2013) odd glial cells. Every neuron is alive and is individually conscious of what it is doing. Most of the trillions of information transmitting synapses are not bipartite (neuron to neuron) but tripartite (neuron, neuron, and astrocyte). The live-neurons synthesize matter like neurotransmitters. "Life" comes in the picture between the software processing of information and 
operation of consciousness. The hardware in the brain is alive! What emerges in the brain is not "life", not "consciousness", but a collective mind, a collective consciousness of trillions of neurons (and glial cells) working under the guidance of self. Why of all the organs in the body, of all other cells of the different systems of the body the neurons have been chosen to create the collective consciousness of the system is an important research question. The property like neuron-philia of consciousness and consciousness-philia of neurons could be part of important research hypothesis in this context, where the non-mitotic "serenity" of genes and the "polarity" of membrane specificities (like cellular membrane prion protein) of the neuron become the focus of scientific investigation.

That brain is not absolutely necessary for memory storage has been shown experimentally in Planarian flatworm (Shomrat \& Levin, 2013) following its decapitation and persistence of the memory despite this! In fact, even in human being a very minimal portion of brain is necessary for awareness. There is a need to re-examine the concept of "minimal brain for identifiable and reportable consciousness", which was originally proposed by Wilder Penfield and Herbert Jasper (1954) on the basis of their vast experience in neurosurgery in epileptic patients. Bjorn Merker (2007) also recently elaborates this. Roger W. Sperry was aware of this phenomenon that the cerebral hemispheres and its commissural systems are not essential for identifiable and reportable consciousness. Even the neural substrate of episodic memory (hippocampus) and affective memory (Amygdale) are not essential for the same. However, the upper brain-stem region (Penfield)/the mesodiencephalic regions (Merker) have been found absolutely essential for such conscious states. In a very recent article, Delafield-Butt and Gangopadhyay while describing the origin of sensorimotor intentionality, emphasised (Delafield-Butt \& Gangopadhyay, 2013) on the "action oriented view presenting brainstem as functionally supracortical though anatomically.... it stands in agreement with Cambridge Declaration on Consciousness...”.

It is yet to be known how this anatomically subcortical structure exercises power over, or overrides the cerebral cortical control in a super-ordinate or supracortical way (Morsella \& Bargh, 2007)! Perrey (2013) has recently considered a possible supracortical location of command for volitional assertion of motor function. A role has also been postulated in biology of shared experience and language development in infants (Trevarthen \& Delafield-Butt, 2013) for anatomically subcortical system, but functionally playing as supracortical.

If ever the operating consciousness for the system to be located, most likely, it would be on the boundary operating across the boundary which in the context of the brain would be supra-cortical in location. There appears to be a near-convergence of views of many open-minded scientists and philosophers on holographic information communication across the evanescent space-time boundary of the system. For a biologist, the system happens to be a cell membrane, the boundary of the individuality in "cell doctrine"! In neurological term, the boundary is the cerebral cortex, the evanescent boundary of brain-to-brain interface. This idea of on-and-across the boundary theory of consciousness could be applicable for all systems that are supposedly to have psyche, irrespective of whether it has or does not have a ganglion or brain! This could be another starting point for developing the science of consciousness. Consciousness is at the centre of this information holograph (Mukhopadhyay, 2012).

\section{Why Consciousness Is Relevant in Science?}

We have not analyzed the issue why consciousness is relevant in science. Can't we continue to do science without this nonsensical consciousness? We probably may get an answer if we address the following five 
issues:

(1) Hit me on my head and I fall unconscious! My "life” continues to run. My "mind” continues to work within: Information processing is being carried out as well. To a painful stimulus even my "self" screams and resents. However as a whole, as a being, I am functionless, I am useless. If I continue like this, soon I would be even a liability to others. As I gain consciousness my "value" to others is altered and I transform from a liability into an asset! Consciousness thus adds "values" to my existence. The role of consciousness can be understood by examining the difference in behavior and expression between unconscious and conscious subjects, also recording how the value of the subject in the society and the subject's sense of values changes with the rise of the level of his consciousness. As the unconscious subject comes back to conscious state, his self, mind, life, and information processing all get placed in their respective slot to function for the whole, independently but still jointly and coherently. This unification of independence of several altogether different sovereign operations maintaining absolute coherence is a function that cannot be accomplished without functional consciousness. Consciousness, thus, makes its great political statement.

If all what have been said is not dismissed as only assumption, the appropriate research question could be a formulation on how consciousness binds all these functionally independent entities. The research hypothesis to this effect could be stated that it is done through suitable change in the geometry of information. In conforming this geometry of information, the element of "life" has a role. The kind of binding we are discussing here is a kind of informational binding. No information is, however, an island and therefore, informational manifold becomes another important role player in this binding.

Consciousness is that by which one looks at what has been going on in one's mind, self, and life! To dissect out this assumption, the research question could be how consciousness that is completely independent of everything else watches and even regulates activity of others. The research hypothesis in this context is that while consciousness participates in the act through operation of life by means of informational binding, also acts as witness of the process by means of a kind of phenomenal binding! This stimulates us to investigate further the distinction between informational and phenomenal binding;

(2) An intelligent system does not have the ability to will: A conscious system has! The principal function of consciousness is volition following a "will". It provokes us to investigate how the degree of freedom of consciousness from its constrain (anchorage with life, mind, information, matter) determines the degree of freedom for its will. Absolutely free consciousness can only have an absolutely Free Will that consciousness executes with absolute ease and pleasure. The "choice", a phase-specific freedom in variant of "will" of the small system merely indicates that it is conscious and operates as a part/nest of the larger whole. On the other hand, lack of lead "political will" can push a nation to the brink of peril.

Readiness potential over the skull, i.e., outside the brain precedes the subjective experience of "will" by several hundred milliseconds (Benjamin Libet’s experiment (1999)). "Will” is translated as volition, which has an intention or purpose, which connects successive moments directed towards the goal. Next follows the planning, for execution of the "will" in "microscopic" (quantum nature) world, which manifests finally in macroscopic (classical nature) world. Therefore, for Free Will to be on the wheel it has to encounter the plane of self-consciousness, plane of volition with intention, plane of planning which requires information rearrangement to serve the intention, the plane of execution in the microscopic world, and its final expression in macroscopic world. This creates a pentaune (five in one) model on how Free Will comes down to Newtonian wheel! The model can be dissected with several research questions and hypotheses at appropriate levels; 
(3) Any conscious system is incessantly confronted with numerous informational and phenomenal inputs from its surrounding environment: This remains an obligatory choice of the "self" of the system for its very survival to reject or adapt with or even to evolve in a new situation loaded with different and often unique information or phenomena. A non-conscious system has nothing to do with such informational or phenomenal inputs. Cognitive closure or the failure of the conscious system in such rejection, or adaptation and evolution will lead it to succumb to such selection pressure and make it evolutionarily stagnant (e.g., crocodile). Consciousness, thereby, is essential for such living and growing which is essentially creative. If this is also considered a statement of assumption one is free to dissect the statement with appropriate research questions and hypotheses in reference to various explanatory gaps documented in the evolutionary biology;

(4) Another important function of consciousness is to grant autonomy to its sub-system that has achieved a reasonable degree of perfection as desirable for running the system coherently as a whole: Once the subsystem achieves perfection it is allowed to function freely, in neurological term as reflex, which not necessarily is to be brought under incessant surveillance of consciousness. Therefore, even the complicated reflex like pupillary reflex, events like binocular rivalry and McGurk effect (hearing lips and seeing voices) (McGurk \& MacDonald, 1976) is not conscious events and are left autonomous to be executed below conscious level! On the other hand, the slightest imperfection in the system is flagged and alarms a call for consciousness. Imperfection requires surveillance by consciousness while perfection is automated within the system. For this reason, autonomy has been conferred to organs like heart, lungs, kidney, and liver but not to the genital organs! In nature, autonomy has been offered even to monsoon but not to tsunami! A couple of research hypotheses could thus be made in the context of human resource management and environmental science;

(5) Consciousness alone is totally inactive: Consciousness's representative in the system is self. Self's tools of operation are mind and intellect. However, bereft of consciousness the mind is not only divisive but is also practically unproductive. This can be supported by evidence emanating from the result of meta-analysis of success or failure of strategies and skills adopted in different kind of management. Consciousness makes sterile mind fertile. Only while in functional connection with consciousness, mind can conceive information and deliver "form" (space time) out of it. Are all these to be considered as statements of assumption? The research question and hypothesis may be formulated on why an intelligent robot cannot have the degree of fertility in conceiving information as it is observed in a conscious system of equivalent intelligent level.

We have just touched on the areas where consciousness might have a place in algorithm of science, especially in psychology, mind-body, and molecular medicine, in adaptation, evolution, and organization of sub-systems, and in management, political social, and environmental science. We can also easily chalk out the role of consciousness in algorithm of pathogenesis of psychosomatic disease or in their remedial measures! The whole discipline of psycho-neuro-immunology/endocrinology remains open for this. Simple research hypothesis like "level of being-consciousness is negatively correlated with incidence of immune disorders" and the "level of being-consciousness determines the frequency, type and the severity of infection" are relevant to ponder with.

\section{Cell's Choice}

\section{The Pentagon of Cell Psychology}

Choice of a cell. Does a cell have any choice over different options it has? Of course, yes! Choice is a function of self. Cell is a self-organizing system. Self is categorically identical to consciousness as such. 
However, the choice of a physiological cell works with very limited number of options:

(1) A differentiated cell has no other choice but to behave in the designed differentiated way. For example, liver cell, kidney cell, heart cell and the neuron or a neutrophil in blood have very little choice other than what they are respectively supposed to do;

(2) A committed stem cell cannot choose beyond its commitment, e.g., committed myeloid progenitor cell develops along myeloid line or lymphoid progenitor cell develops along lymphoid line;

(3) Non-committed stem cell can differentiate into multiple directions depending on the microenvironment the cell has been allowed to grow. When a stem cell from the brain-marrow is put in HIM (Hemopoiesis Inductive Microenvironment), it grows into blood cells. Vice versa is also true. Each totipotent cell of a zygote can give rise to a full embryo!

The principal constraint in all of three situations is the constituent genes of the cell.

When we accept that the cell has a choice, it is also implied that the cell has "thinking machinery" and a repertoire of "memory" of experience on the basis of which it can choose. This memory of past experience would be second strong constraint for choice. Richard Dawkins (1976) describes the unit of "thought" as "meme" (rhyming with genes). Self, memes, and genes are involved in the choosing process. Two other factors are also involved in the decision-making. One works from behind, the ground consciousness, and the other on the front, the challenging information. Information is the common currency for business transaction by and between self, memes, and genes.

Self, memes, genes, information, and consciousness are five factors involved in the decision-making process of a cell and in its choice. We may call this decision-making body as the "Pentagon of Cell". More about interrelationship of self, memes, genes, information, and consciousness could be found out from author's previous publication (Mukhopadhyay, 2010).

Supremacy of "self" over the memes and genes. Let us examine a famous quote of Richard Dawkins,

We have the power to defy the selfish genes of our birth and, if necessary, the selfish memes of our indoctrination. We can even discuss ways of deliberately cultivating and nurturing pure, disinterested altruism—something that has no place in nature, something that has never existed before in the whole history of the world. We are built as gene machines and cultured as meme machines, but we have the power to turn against our creators. We, alone on earth, can rebel against the tyranny of the selfish replicators.

We might begin with the question: Who are we? In response, we land up with "self". We may, therefore, be excused for a small alteration in the quote, as felt needed here. Memes and genes are not our creators, but they are ingredients in individuation. Consciousness is our creator and by the supremacy of our self, "We, alone on earth, can rebel against the tyranny of the selfish replicators". But, the question remains: How does this supremacy of self become so natural? Most likely, this is brought out by virtue of the difference in category of information self, memes, and genes carry are sensitive to! It is by this specific category of information only, memes and genes overpower self. And, it is this information, which establishes supremacy of self over memes and genes. An uncomfortable self in search of freedom tries to come out of the trap of memes and genes. If it could successfully "connect" itself with the unconditional consciousness-as-such, it could restore its natural supremacy. And, this connection is established by means of a special category of information. In spiritual practice it is done through "Mantra", which carries information on the Divine.

The paradigm shift. The argument has been built up to convey the message that information is more fundamental than space time and energy, self, memes, and genes and, therefore, entails a paradigm shift where 
the power is not with the genes or memes, or even with the self but with Information. The genes and memes neither generate information nor use information. It is information, which uses genes and memes as means for materialization of its content and intent.

Psyche of a cell. There is another way of looking into this pentagon of cell psychology. Psyche is non-observable but influential. As "non-observable influential" psyche influences the behavior at observable level! Psyche, contrary to what is popularly conceived, is not monolithic. Integral components of psyche are consciousness, mind, self along with its motivational facets, life-principle, and information. The Composite Model of Psyche was presented by the author in The World Congress of Psychotherapy in Buenos Aires, Argentina in 2005 which was subsequently published on the same year (Mukhopadhyay, 2005). Self's motivational facets are imprints of elementary phenomena involved during creation of self from consciousness-as-such.

Most likely the organelle development in a cell has followed the line of five elementary phenomena: (1) Cell membrane could be the representative of the elementary phenomenon, love; (2) Nuclear replication apparatus could represent the elementary phenomenon, sex; (3) DNA-transcription apparatus with mRNAs and ribosomes, etc., might represent the elementary phenomenon, life; (4) Lysozome representing the elementary phenomenon, ego; and (5) Mitochondria represent the elementary phenomenon, death. Mitochondria work incessantly producing energy to fight against death. Caspase pathway is within the mitochondria. Heme synthesis pathway travels through mitochondria. Cortisol synthesis pathway also travels through mitochondria. This extraordinary organization of five important organelles within the cell makes the decision making body of the cell, the Pentagon of Cell psychology. This concept of pentagon of cell-psychology is originally available in author's work, The Dynamic Web of Supracortical Consciousness (Mukhopadhyay, 1987). The author presented the same theme in The World Congress of Pathology in Madrid, Spain, in 1992.

\section{Phenomenal Role of Microtubules}

What are microtubules doing then? Hameroff and Penrose's theory on consciousness revolves entirely on microtubules. Are the microtubules then the actual "Pentagon” where decision is made?

In response to this question raised it may be said that microtubules are responsible for integrating five phenomena of elementary phenomenology within a cell. At the observable level microtubules integrate the functions of five important organelles within the cell.

Microtubules are part of cytoskeleton. Along with microfilaments they form the skeleton of the cell. Because of activity of cytoskeleton, actin-myosin sliding over each other we can move, our heart pumps even without fatigue. By its anchorage with the cell membrane cytoskeleton maintains the shape of the cell. The required shape change of a cell while passing through narrow tunnel is also executed through cytoskeleton. This cytoskeleton crumbles in Alzheimer's disease resulting in dementia. In alcoholics, the cytoskeleton of liver cell crumbles to form Mallory hyaline. Many anesthetic drugs work through microtubules. Therefore, this "skeleton" of cell has become so important in consciousness study. In 3-D image analysis of a live cell, microtubules are just wonder!

(1) From architectural and civil engineering point of view: Microtubules are architectural marvel with fantastic structure, design, supply, and drainage system. Structural biologists with techniques like crystallography have been working on it;

(2) From mechanical engineering point of view: The cytoskeleton helps in maintaining the appropriate 
spatio-temporal relationship of all five important organelles. They do not allow lysozome to fall upon nucleus or mitochondrion to touch nucleus injudiciously. Microtubules direct chromosomal segregation during cell division (sex). Organization of microtubules in the interphase is guided by centrosome (a pair of centrioles), an evolutionarily conserved microorganelle. Even the mechanical engineers can learn a lot from this;

(3) From IT engineering point of view: The microtubules are structured for information capturing and information storage;

(4) From Electronic and communication engineering point of view: Microtubules are the communicating channels between cell membrane, nucleus, lysozome, and mitochondria;

(5) From biophysical point of view: Microtubules are responsible for integration of the "whole" within the cell. Conformon maintains conformity with the whole. Signal transduction involves Photon and Phonon. Openness of the system for learning and maintenance of perfection is because of neutrinos. An extra-ordinary constellation of conformon, photon, phonon, and neutrino around "self" forms the basis of integration mechanism.

\section{Three Theories of Consciousness in a Living System}

Consciousness is not generated from these microtubules or from the cell membrane. Nor it is generated by the activity of a huge synaptic network. In general, nothing generates consciousness. All three, however, participate in development of conscious experience. In a unicellular organism there are microtubules and there is no context of cell-junction or synapse! The microtubules within the cell, as described above in the perspectives of consciousness, are responsible for integrating elementary phenomenology as well as functions of five important organelles in a cell at several levels: mechanical, electronic, informational, and biophysical levels. Microtubules are the seat of central informational management centre.

Since information is close to consciousness, importance of microtubules in the context of cell-consciousness remains so high! In a bottom-up approach it is information, which connects matter with the mind. In the same vein, it is life-principle, which connects mind with consciousness. When the life-principle ceases to operate, all anchorages of microtubules on the cell membrane start loosening, and orchestrated reduction of OR (objective realty), also stops.

Further, within-cell information-consciousness is not sufficient to explain the whole of consciousness and conscious phenomena. Cell interacts with its non-cellular environment as well as with other cells. A single cell, paramecium, communicates solely with environment. There is no context of synapse or cell-to-cell junction in paramecium case.

As has been suggested by the author since 1987 and is also recently conceptualized by Bruce Lipton in his book Biology of Belief (2005) that conscious interaction between the cell and its non-cellular environment happens across the boundary of the cell membrane, the boundary of individuality in "cell doctrine". The research question is how nonlocal influential(s) operationally connect with local observables within the system to bring measurable change in the behavior of the system. And, research hypothesis is that it is done through holographic information transfer across the boundary of the system.

Conscious interaction between different cells happens through cell-to-cell junctions (the cell-junction has microtubular anchorage) and through synapses in case of neurons (microtubules extend almost up to synaptic membrane). This connectivity is certainly important for networking amongst the group of cells. Connectivity between several millions of units through association, networking, and different levels of communication is 
very much necessary for collective consciousness of the whole.

Therefore, all the three themes: (1) supra-boundary and across-the-boundary theory of consciousness; (2) microtubular theory of intracellular consciousness and conscious phenomenology within the cell; and (3) the synaptic theory of conscious activity in a magnificent neural network, have their respective contribution and are important in a complex tissue, organ and in the organism as a whole.

\section{Consciousness in Physical Science}

Also, another bigger question is what could be the role of consciousness in any of the algorithms of material/energy science. To make any statement in this area or even to pass judgment on possibility or impossibility of having research question seems most difficult. We have stated that this area would be clearer once we know more about "information" and "life”. Is "life" all-pervading in the universe(s)? Is our universe self-aware? Has "life" originated from "matter" or, has "life" been there all through, and has matter originated from life? Such general questions have repeatedly arisen in scientific mind (Mukhopadhyay, 2014). At this stage, five important issues could be stated for further examination: (1) Does consciousness or its companion life-principle, or its representative "self" have a role in bringing order out of chaos, which in present science has been left as "spontaneous"; (2) Can inclusion of nature-consciousness in the scientific algorithm solve the problem of number of dimensions in space-time physics particularly in String Theory and Quantum Loop Theory? Dimension means specific space-time geometry, which is associated with the context generation for information. Strings are advocated at the deeper recess of quantum nature. They are, however, still within Planck's scale of measurement and therefore are suitable conduit to be the vehicle of information from the world, which is beyond Planck's scale! According to several physicists Quantum loop theory is space-time formation independent of the background, while String theory deals with space-time that is dependent on the presence of a background; (3) Does consciousness or life as life-form play a role in transformation of nature beyond Planck's scale into nature within Planck's scale of measurement? Is consciousness/life-form someway involved in connecting dark matter/dark energy with visible matter/energy? (Mukhopadhyay, 2012; 2014); (4) What is the role of consciousness in instantaneous information transmission at a distance and communication in a holographic manner? Can an information holograph function in absence of consciousness? Probably a holograph at classical level always does so! A quantum holograph also can do so selectively. However, for information holograph consciousness seems essential; and (5) If consciousness is actually operational in all physical processes, the prediction (Mukhopadhyay, 2008) is that mind-like structure and process in nature (quantum fields as messenger of infinity) can split information into "form" (space and time) and energy! The energy is obviously information-based, in contrast to matter-based energy we are familiar with. In human situation, information splitting is supposedly a regular phenomenon in a conscious brain! Here arises one great possibility! We are aware that invention of computer represents externalization of some of the properties of mind! Is it possible to develop an information-splitting machine and harness information-based energy? Will this prediction come true? A lot depend on this prediction for consciousness to leave its revolutionary imprints on material/energy science!

\section{Research Questions in Building the Bridge Between Science and Spirituality}

While science deals with the nature, spirituality deals with the nature of consciousness. Within the nature of consciousness, the events are simultaneous, continuous, and often identical. In fact, 
when one identifies simultaneity, continuity, and identity of events, it is worth remembering that in these events there is signature of consciousness.

Within the nature, which the present science investigates, the events are non-simultaneous, discontinuous, and non-identical. Einstein's constant (velocity of light) excludes simultaneity of events. Planck's constant excludes continuity of events and Entropy barrier excludes identity of events. Under the umbrella of these three constants present science continues to work.

Therefore, the statement, "as above, so below", is not exactly true. The issue in science-spirituality horizon is how to connect such events which are "above" and which are "below". There is a problem of boundary between the nature present science investigates and the nature which spirituality presents. There is a boundary effect!

What is the mechanism of "transformation" of "above" into "below"? Is it a mirror-effect distinguishing reality from its virtual image? Or, is it Plato's allegorical cave with shadow show of a hidden reality behind? Is it merely an inversion with an upside-down effect in two or three dimensions? Or, is this boundary effect an inside-out phenomenon, which is multi-dimensional? These are some of the research questions in science-spirituality horizon!

\section{The Boundary}

What is this boundary that distinguishes "above” from "below”? In popular sense, "above” means "heaven" and "below" connotes earth. What separates heaven from earth is the phenomenon of death! Separation of heaven and earth is a phenomenal separation! In the heaven, "love” is "life". The earthly events are motivated by "ego" and "sex". "Death" stands in between the heaven and the earth! Love, Life, death, ego, and sex are five motivational facets of self. Self having taken its motivational facets into confidence can conquer the boundary!

In the living state, what distinguishes "above" from "below" is the barrier of mind. It is mind, which distorts "above” into "below". Sri Aurobindo, the 20th century's accomplished mystical philosopher from India, found solution of such dilemma in what he called "supramental"!

When expressed in a totally objective way in third person's perspective, this boundary could be stated to be the boundary of the universe or the boundary of a functioning brain. The boundary of the universe is between ZPE (Zero-Point-Energy) and EM (the essence from which the system of multiverse is generated). In the context of the brain, the boundary could be found between cerebral cortical activities and supracortical nature.

Based on the above-mentioned several assumptions, research questions, and research hypotheses could be formulated to investigate the horizon of material science (horizon issues) and the horizon of nature of consciousness.

\section{The Gravity, Quantum, and Mind}

\section{Physics and Psychology}

Space can be quantized. Time can be quantized. But quantization cannot be done of the warped spacetime. It requires the signature of a genius of Einstein to be accepted in science that warped spacetime is gravity. Nothing moves in spacetime. Gravity and spacetime are force. Energy could be quantized. Could the force be so? This situation probably made Einstein averse towards the concept of "discontinuity" ingrained in quantum 
physics! He did not accept the possibility of the presence of a particle called "graviton" mediating gravity. By the by, gravity is the weakest of all the four.

What is this gravity for the nature? As the feelings of human psyche could be expressed in following four ways, emotional/visceral (limbico-hypothalamic), intellectual (limbico-cortical associative), mystical (limbico-cortical-prefrontal decisive), and executive (limbico-cortical pyramidal and extrapyramidal), similarly four forces of nature namely gravity, weak force, strong force, and electromagnetic force, are nothing but four ways of expression of feelings of the "psyche" of the nature. As it is of no use to investigate the common source from which psychological feelings are expressed, similarly it will be unrewarding to look into the possibility of a common source of four forces of nature as super force. Based on these arguments, the possibility of having quantum gravity is a remote dream for science even in the 21st century without involving components of psyche in the process.

According to the author, there is one kind of "energy", which cannot be quantized. This non-quantizable energy remains in association with gravity. This is what is called dark energy! This energy, in contrast to energy radiated from black body, is not emitted as quantum. In this sense, one encounters two kinds of energy: quantizable and non-quantizable. Quantizable energy is within Planck's scale of measurement. Non-quantizable energy is not! Their possible relationship has been described in author's published article, "From god particle to consciousness....” (Mukhopadhyay, 2012).

According to the author, discrete space, discrete time, and non-quantizable dark energy are generated from information, delivered by mind or mind-like process or structure in nature (Mukhopadhyay, 2008). However, it is difficult to understand how within the ambit of "information", space, time, and non-quantizable energy remain accommodated! It is not clear whether all remain as discrete space and discrete time or as warped spacetime. What is the relationship between gravity and mind (or mind-like structure and process in nature) is one of the most relevant research questions in the area.

For the senses, space is only 3-dimensional. According to Kaluza-Klein hypothesis, space is 10-dimensional. Where are the rest seven dimensions? Are those in scientist's mind? Mind in higher psychology is considered as independent “sense organ”. Dimensions are spacetime constructs. And, spacetime constructs are products of mind! However, many of the dimensions have mathematical foundation. Abstract mathematics is one of the products of evolved cognitive mind!

\section{Psychology and Physics}

Life, gravity, and mind. Psyche is not monolithic. It is a polylithic structure and process which is consisting of five entities; namely consciousness, mind, self along with its five private facets, life-principle, and information. How this psyche as a whole, or some of its constituents are related to space, time, and gravity?

Is self related anyway to gravity? Self's movement is unrestricted in spite of its conditioning by information and "memes". Self can move from any nest to another of nature-consciousness. Self moves from one universe to another. Self is a traveler across multiple universe(s). The boundary does not exist for self. In physics, the hypothetical particle, "graviton" which is the mediator of gravity is assumed to be the communication carrier between multiple universe(s). "Graviton" moves across several universe(s). Many members of the science-spirituality group (particularly the followers of T. D. Singh of Bhaktivedanta Institute) believe that particulate form of consciousness is "spiriton" (rhyming with photon, phonon etc.) which has unrestricted entry anywhere. In the context of the gravity, it would be interesting to find out the relationship 
between "graviton" and self (and spiriton), if any.

Gravity can be overcome only with the help of life-principle. Plants grow against gravity. Birds fly against gravity. Fish swims upstream against gravity. Against gravity, we climb stairs and mountains and conquer Mt. Everest. In dream, often one swims in the air (wondering body experience) against gravity, penetrates through the barrier of a wall, which is representing the situational gravity. In out-of-body experience, self circumvents gravity. During psychedelic experience, one feels "lighter" than one's actual weight. During deep TM (transcendental meditation) one is often reported to levitate!

The scientists of CERN laboratory have recently shifted their focus to find out the "particle" mediating antigravity. Most likely, the conclusion of the research would lead us to a relationship between anti-gravity particle and life-principle, which might be termed the "lifetron" (rhyming with electron, in the language of Paramahansa Yogananda)!

In folk language, we assess the "gravity" (warped space time) of the situation (space time format) with mind! As an example of extraordinary mental feat or a feat of self, a strong mind or self can even interject on the moon-ocean tide interaction, which is mediated through gravity.

How much of space and time will be warped as gravity, how much of them will be utilized for structurization of form/image/archetype, and how much of this will remain as quantizable discrete space and time are done through mind or mind-like structure and process in nature, but scrupulously determined by life-principle. Mind owes its strength from its connection with life-principle.

Without the help of gravity, life is not situational and cannot grow robust in four dimensions. The relationship between Higgs-boson, which is elusive at material level and life-principle, which is elusive in non-local level, is relevant in this context (Mukhopadhyay, 2012). Biologized gravity does add "charm" to the personality and contributes its expression in aesthetics. On the other hand, without the ability to overcome/circumvent gravity voluntarily life will be evolutionarily stagnant, as it has happened in cases of crocodiles. In absence of operation of life-principle, gravity engages in construction of black hole, expressed to the senses as the phenomenon of "death".

\section{Concluding Remarks}

A number of new ideas have been introduced in the form of assumptions and research hypotheses on epistemic consciousness. In the concluding part, it is asked how to approach consciousness as an ontological entity. What is consciousness sensitive to for getting a response from it? Mind is known to be sensitive to information. Self is sensitive to phenomenon. Consciousness is not seen to respond to any known kind of stimulus, may it be energy, information, or phenomenon! Consciousness as ontological entity, in contrast to mind, self, life, and information, is immutable and an inviolable constant that operates in silence stillness emptiness and nothingness. This invariant is not man-made and does not kow-tow to any other entity in nature. Consciousness, as ontological entity, is understood to be sensitive to the process of disarmament of conditioned properties! This process of willful complete unconditional surrender melts consciousness. It not only responds but also is compelled to operate on the issue presented to it. To make ontological consciousness epistemic, therefore, the self of the system has to lay down its properties to consciousness. How consciousness asserts its supremacy over self, life, mind, information, and matter by stripping off their conditioned properties and dressing them up as and when necessary seems the most difficult question for science. 


\section{References}

Chen, W. G., Chang, Q., Lin, Y., Meissner, A., West, A. E., Griffith, E. C., Jaenisch, R., \& Greenberg, M. E. (2003). Derepression of BDNF transcription involves calcium-dependent phosphorylation of MeCP2. Science, 31, 885-889.

Dawkins, R. (1976). Memes and genes. Retrieved from http://library.thinkquest.org/C004367/ce2.html

Delafield-Butt, J. T., \& Gangopadhyay, N. (2013). Sensorimotor intentionality: The origins of intentionality in prospective agent action. Developmental Review. Retrieved November 5, 2013, from http://www.sciencedirect.com/science/article/pii/ S0273229713000403

Epley, N., Converse, B. A., Delbosc, A., Monteleone, G. A., \& Cacioppo, J. T. (2009). Believers' estimates of God's beliefs are more egocentric than estimates of other people's beliefs. Proceedings of the National Academy of Sciences of the USA (Vol. 106, No. 51, pp. 215333-215338). Retrieved November 5, 2013, from http://www.ncbi.nlm.nih.gov/pmc/articles/PMC27874 $68 /$

Ganti, T. (2003). The principles of life (With a commentary by Eors Szathmary and James Griesemer). Oxford: Oxford University Press.

Kandel, E. R. (2013). From nerve cell to cognition: The internal representation of space and action. In E. R. Kandel, J. H. Schwartz, T. M. Jessell, S. A. Siegelbaum, \& A. J. Hudspeth (Eds.), Principles of neural science (5th ed., pp. 370-391). New York: Mc Graw Hill.

Libet, B. (1999). Do we have free will? Journal of Consciousness Studies, 6(8-9), 47-57.

Lipton, B. H. (2005). Biology of belief: Unleashing the power of consciousness, matter and miracle. Carlsbad, California, New York: Hay House, Inc..

McGurk, H., \& MacDonald, J. (1976). Hearing lips and seeing voices. Nature, 264, 746-748.

Merker, B. (2007). Consciousness without a cerebral cortex: A challenge for neuroscience and Medicine. Behavioral and Brain Sciences, 30, 63-134.

Metzinger, T. (2007). Self models. Scholarpedia, 2(10), 4174.

Morsella, E., \& Bargh, J. A. (2007). Supracortical consciousness: Insights from temporal dynamics, processing-content, and olfaction. Behavioral and Brain Science, 30(1), 100.

Mukhopadhyay, A. K. (1987). Psychology of a cell—A holistic concept. In The dynamic web of supracortical consciousness (pp. 156-166). New Delhi: Conscious Publications.

Mukhopadhyay, A. K. (2005). A composite model of the psyche: Its practical relevance in psychotherapy. In G. Shankar (Ed.), Psychotherapy, yoga and spirituality (pp. 20-29). New Delhi: Jagdamba Publishing Company. Retrieved from http://akmukhopadhyayconsciousness.com/pdf/LINK1.pdf

Mukhopadhyay, A. K. (2008). A radical view of information: On its nature and science. Frontier Perspectives, 16(2), 19-29. Retrieved from http://akmukhopadhyayconsciousness.com/pdf/LINK6.pdf

Mukhopadhyay, A. K. (2010). The self and its memes and genes: Genes, memes, self (brain), information and consciousness. In P. Sengupta (Ed.), History of science and philosophy of science: A historical perspective of the evolution of ideas in science (Project History of Indian Science, Philosophy and Culture (PHISPC), Vol. XI, Part VI, Chapter 21, pp. 481-558). New Delhi: Pub. Pearson. Retrieved from http://akmukhopadhyayconsciousness.com/pdf/LINK8.pdf

Mukhopadhyay, A. K. (2012). God particle to consciousness: Life-science, neuroscience and nonlocal science hold the key. In S. C. Mishra, S. Ghosh, \& V. Agarwal (Eds.), The science and spiritual quest: Integrating capabilities with values. Proceedings of The 7th AISSQ Conference, Bangalore, Bhaktivedanta Institute, Kolkata, India (pp. 108-123). Retrieved from http://akmukhopadhyayconsciousness.com/pdf/LINK12.pdf

Mukhopadhyay, A. K. (2012). Information holograph: The structure, the source and its operation. International Journal of BioEngineering, NeuroSciences and Technology, 2(2), 12-32. Retrieved from http://akmukhopadhyayconsciousness.com/pdf /LINK13.pdf

Mukhopadhyay, A. K. (2013). Non-observable influential(s) in the domain of consciousness: Their identification and description. Psychology Research, 3(11).

Mukhopadhyay, A. K. (2013). Setting the agenda for a Science of information. Retrieved from http://akmukhopadhyayconsciousness.com/pdf/LINK14.pdf

Mukhopadhyay, A. K. (2014). "Life” wthin the Akhanda Worldview. Dialogue and Universalism (in Press).

Nagel, T. (1993). What is the mind-body problem? Ciba Foundation Symposium 174-Experimental and Theoretical Studies of Consciousness, UK, John Wiley. 
Penfield, W., \& Jasper, H. H. (1954). Epilepsy and the functional anatomy of the human brain. Boston: Little Brown and Company.

Perrey, S. (2013). Promoting motor function by exercising the brain. Brain Sci., 3, 101-122. doi:10.3390/brainsci3010101

Shomrat, T., \& Levin, M. (2013). An automated training paradigm reveals long-term memory in planarians and its persistence through head regeneration. The Journal of Experimental Biology, 216, 3799-3810. doi:10.1242/jeb.087809

Sperry, R. W. (1987). Structure and significance of the consciousness revolution. J. Mind Behav, 8, 37-65.

Stapp, H. P. (2005). Quantum theory of the human person. In A. Elitzur, S. Dolev, \& N. Kolenda (Eds.), Quo vadis quantum mechanics? (pp. 397-404). Springer, Berlin Heidelberg.

Trevarthen, C., \& Delafield-Butt, J. (2013). Biology of shared experience and language development: Regulation for the intersubjective life of narratives. In M. Legerstee, D. W. Haley, \& M. H. Bornstein (Eds.), The infant mind: Origins of the social brain. New York: The Guilford Press. 